\title{
Learning from 35 Years of Community Child Health Services in the United Kingdom: Reflections for Community Geriatricians
}

\author{
Fiona Finlay $^{1 *}$ and Simon Lenton ${ }^{2}$ \\ ${ }^{1}$ Consultant Paediatrician in Community Child Health, UK \\ ${ }^{2}$ Retired Consultant Paediatrician, $U K$ \\ *Corresponding author: Fiona Finlay, Consultant Paediatrician in Community Child Health, UK
}

Submission: 非 November 19, 2018; Published: 眥 November 27, 2018

\begin{abstract}
This article reviews the development of community child health services in the UK over the last 35 years and the role of the community paediatrician and reflects on the issues that had to be addressed to enable the specialty to evolve to the service that it is today. The article considers three areas-clinical practice, local service delivery and the national issues of leadership and policy, all of which may be relevant when developing community geriatric services. Community paediatrics and community geriatrics share many common aspects including increasing numbers of patients, the complexity of providing care for patients with multiple comorbidities, the issues of consent and confidentiality and the involvement of the extended family in care plans.

At a local level organizing clinical care in the community poses many challenges not present in a hospital setting where the multidisciplinary team, investigations and specialist care are all based together. This requires careful organization of time and resources to provide practical holistic care for individuals with complex needs in a number of settings. Developing meaningful working relationships with primary care and hospital teams, as well as with social care and other agencies involved is essential for success. A local champion is often required to negotiate new roles and responsibilities and local commissioning support is required for the development of a competent multiagency team that fits seamlessly between primary care and hospital care. Long-term leadership at a national level is essential to develop relevant health service policy, workforce estimates and training competencies, without which there will be insufficient numbers of appropriately trained geriatricians to work in community settings.
\end{abstract}

For the long-term viability of both paediatric and elderly care teams in community settings the clinical research base and the health service research base requires investment in order to produce the evidence required for future service development and improved outcomes for patients.

Keywords: Community geriatrics; Community paediatrics; Community child health; Integrated care; Multimorbidity

\section{Introduction}

The medical specialties of geriatrics and paediatrics have followed similar chronological developmental courses, with older people and children being recognized as vulnerable groups in society during the Victorian era and with subsequent service provision initially focusing on acute conditions managed in hospital settings and community-based provision developing more recently $[1,2]$. Paediatricians and geriatricians rarely talk to one another about service delivery in daily clinical practice even though their client groups share many characteristics. Both paediatric and geriatric services recognise the importance of integrated holistic care and have developed child-friendly and elder-friendly guidelines $[3,4]$ and both have national service frameworks $[5,6]$ to guide the development of services in the UK. With changing epidemiology and a higher prevalence of long-term conditions, health policy in the UK is encouraging more out-of-hospital integrated, multidisciplinary care, with a focus on prevention for both children and the elderly [7-9]. Child health provision, delivered by community-based multidisciplinary teams including community paediatricians, has been in development over the last 35 years and this article reflects our personal experience of service evolution during this time and highlights some lessons which may be helpful for those working in community-based elderly care teams including community geriatricians.

The authors have had a wide breadth of experience in community child health ranging from the care of children with a range of clinical conditions, safeguarding, disability and palliative care as well as experience in public health, health policy and NHS management. This article will focus on three aspects - clinical practice, the development of local services and then national leadership and policy issues. While written from a perspective within the UK, many of the issues raised will be applicable to health services in other nations and the intention is to reflect and discuss issues pertinent to community geriatrics. In both services it is unlikely that there is a single universal model of service 
provision which is ideal in all communities, but the incorporation of values derived from human rights, evidence from research and a commitment to learning through quality improvement should improve service delivery, health service outcomes and quality of life for patients.

\section{Similarities}

Morbidity is increasing within both specialties - the elderly are living longer often with multiple comorbidities and children are surviving into adulthood with increasingly complex problems, for example, those who were born extremely prematurely, those with underlying genetic and metabolic conditions and following treatment for cancer[9]. There are also many children with new morbidities who are best supported in community settings rather than a hospital environment, for example, children and young people with attention deficit hyperactivity disorder (ADHD), autistic spectrum disorder (ASD) and mental health problems. Both specialties are struggling to find the optimal arrangements to provide services for those with multiple morbidities $[10,11]$. A child with cerebral palsy may need to see a neurologist for epilepsy, orthopaedic surgeons for scoliosis and joint contractures and a gastroenterologist for nutrition and gastrointestinal problems whilst simultaneously requiring support at school from a range of education specialists. Their family may also require services at home to help with the burden of care including feeding, sleeping and toileting. A similar package of care may be needed for someone with dementia who may also have hypertension, diabetes, and a stroke or Parkinson's disease, coupled with other age-related deteriorations in physical functions [12]. Both children and the elderly are dependent on their families for support. The nature of extended families is changing in the UK - families generally have fewer children, grown-up children often live far from their parents and there are more reconstituted families. Both families with children and the elderly suffer disproportionately from poverty and inequalities [13]. The concept of an extended family responsible and caring for vulnerable family members is diminishing, while institutional organisations such as care homes and other residential options are on the increase for the elderly and more out-of-home care provision is available for children as a greater proportion of parents both work.

The influence of public health is changing - in the past the focus was on improving housing, sanitation, nutrition and working conditions. Today new models of public health are required to tackle issues such as quality of food, the impact of social media or loneliness and mental health. In both paediatric and geriatric specialties it is essential that the health service elements of public health programmes are delivered by competent practitioners with appropriate training, to ensure equity of health and prevention of problems throughout the populations served. The human rights of both children and the elderly have been recognised with national policies to protect and promote well-being enacted in society and within health service provision. In both age groups abuse has been increasingly recognised and safeguarding services developed to detect and protect the most vulnerable [14-16]. Both community geriatric and community paediatric services demonstrate considerable variation in performance, function and outcomes across the UK [17]. While some of this variation is a reasoned response to local circumstances such as rurality, deprivation, geography and population or health community size, some is more difficult to explain and requires further exploration.

Learning from community child health - clinical practice: The first newly appointed consultant community paediatricians in the early 1980s had trained in hospital settings but now found themselves practicing in a range of community settings not always suitable for delivering high-quality clinical care, for example in schools, family homes and respite care settings, which did not have privacy, examination couches or adequate lighting. Clinicians often carried patient notes, basic clinical equipment, weighing scales, a child development kit, a selection of referral forms and a dictaphone. For the community paediatrician the focus of their clinical work changed from acute work practiced in hospital settings to the day-to-day management of long-term conditions and prevention of comorbidities, working closely with other members of the multidisciplinary team and primary care teams. Within a hospital members of the team meet regularly, both formally and informally, to discuss clinical care and concerns, whereas in the community teams must organise themselves in ways so they may come together to provide an effective, efficient and equitable multidisciplinary service for patients. In community child health teams discussions focus on treatment options and quality of life, with participation from the child encouraged. Many meetings involve the family such as "team around the child meetings" or "family group conferences". Whether this is an appropriate model for elderly people remains to be explored fully [18]. As young children develop into young adults they become more autonomous and the locus of control and decision-making passes from parents to the young people themselves. Assessment of competence and confidentiality are important elements of decision-making. Some elderly patients become less cognitively able with time and the best interests of the individual must be advocated by the clinician with active involvement of other family members.

Learning from community child health - local service delivery: Local service delivery focuses on the practicalities of developing, establishing and improving local practice. The challenge can be expressed in terms of doing the right things, to the right people, with the right staff, in the right place, at the right time, with the right support, to achieve the right outcomes all at the right cost. To achieve this is surprisingly difficult and requires the engagement of a wide range of organisations and professional groups and rarely are good clinical skills alone enough to achieve this aspiration [19]. Organizational management training for clinicians may therefore be important to achieve local change. Defining the role and the benefits of a new service and then communicating clearly with other providers and teams is essential for success. The evolution of a new service often challenges the ways that traditional services have been provided, for example, clinic appointments in the acute paediatric sector usually last for 10-20 minutes, however children 
seen in the community with long-term complex conditions may require much longer appointments, ideally with people who know them well, for example, staff from education settings, in addition to their parents. Therefore, moving clinics from hospital to special schools makes sense but organizationally may be difficult to achieve.

Follow-up consultations are frequently undertaken on the telephone rather than face-to-face. Developing community child health nursing teams to enable children with long-term conditions and life-limiting conditions to stay at home rather than visit hospital is also highly valued by parents, but it took time to achieve funding and establish this way of working. Palliative care may be delivered at home while hospices are frequently used for respite care in addition to terminal care. Community-based palliative care for elderly people is beginning to evolve [20]. Clarity about specialist provision is important. Originally in community child health consultants had generic roles and supported primary care by taking all referrals that were unlikely to require hospital investigation or treatment. As more consultants were appointed, they developed specialist interests in a range of conditions including physical disabilities, sensory disabilities, audiology, behavioural issues and palliative care, whist others developed expertise in safeguarding, adoption and fostering, and public health programmes, with others taking on the role of immunisation coordinator. Today some consultants do both generic and sub-specialty work, whereas others only do sub-specialty work. There are advantages and disadvantages of specialization - it should improve the quality of care in the specialist area but may undermine service provision in other areas such as providing an on-call rota for child protection and child deaths. Similar debates around the degree of specialization within community geriatrics is inevitable - should all patients with dementia or Parkinson's disease be under the care of an individual subspecialist or team? Over time community child health services have evolved and as the role of community paediatricians have become more widely accepted training and workforce development programmes have had to adapt accordingly. In the early days of community child health consultants were expected to participate in the hospital out-ofhours on-call rota equally with their acute colleagues and while this may have been appropriate in small departments, in larger places it became difficult to participate in acute service activities as well as managing community commitments. Likewise, some consultants had admission rights with ongoing clinical responsibility for their patients which at times conflicted with community clinical commitments. Some developed a system where there was both a hospital paediatrician and a community paediatrician responsible for a geographical area, the hospital paediatrician providing acute care and the community paediatrician ongoing care for those with long-term conditions, the consultants working closely together.

To deliver an effective service it is essential to have access to an appropriate clinical environment supported by the necessary infrastructure. Many community paediatricians see children in primary care settings as families appreciate being seen in a familiar local environment. Often there are opportunities to discuss patient management with the primary care team, possibly reducing the need for consultant follow up. Another important role of secondary care teams is to support primary care teams through education, training, face-to-face meetings and joint clinics, which is facilitated through working in primary care settings. The concept of child health general practice harbours have been promoted nationally [21]. Changes in demography, morbidity, clinical evidence and multidisciplinary working determine clinical workloads. The introduction of market principles into the NHS initially resulted in a purchaser-provider split, which then evolved into a commissioning role with public, private and voluntary sectors competing for contracts to provide components of health services. Commissioning support is important for service development, stated simply, if not commissioned it will not develop. Commissioning care for patients with complex conditions and multiple comorbidities is extremely challenging and integrated care, while ideal, is often difficult to achieve. It is important to work with commissioners and to be able to demonstrate efficiency, effectiveness and equity, together with safety and good patient experience. This is probably best demonstrated through systematic continuous quality improvement. Failure to engage with commissioners and undertake audit cycles and quality improvement projects may lead to poor service development resulting in inadequate clinical services for patients and stress for staff.

Providing holistic care for children in the community involves more than merely health services. An understanding of other agencies in the public, voluntary and private sectors is required, along with active engagement, remembering that it is important to focus on the patient and their needs rather than territories of organisations. The development of care pathways for common paediatric conditions has been a useful process, defining the component parts which need to be in place and working well together to achieve optimal outcomes. More recently there has been interest in integrated providers who can provide care covering the whole pathway, delivered by teams working within a network [22]. Generally, the component parts are delivered by teams of professionals who then work collectively with other teams creating a network of integrated care in a geographical area. Good relationships between commissioners and teams working in networks is essential for incremental improvement in outcomes [23]. Developing a new community-based specialty which complements existing local services requires a consensus across a wide range of professional groups covering both primary, secondary and tertiary care, with practical examples of how this can improve outcomes for patients. Management roles may not necessarily fall to medical practitioners, but this is often the case and while leadership and management are different, nonclinical expectations must be defined and commissioned in order to facilitate the development of a new service. Not all clinicians are prepared for management and leadership roles and further continuing professional development will be required. Unlike hospital hierarchies where tradition has often established patterns of behaviour and accountability, the development of community teams may require more negotiation and collaboration. 
Learning from community child health - national leadership and health policy issues: Policy can be enacted in many different ways ranging from legislation (and regulations to interpret legislation), resource allocation including fiscal measures, public education, professional education (including curriculum development and professional development), organizational change, research priorities and quality improvement. Most are relevant to the development of a new medical specialty. The importance of national leadership when developing a new specialty cannot be overstated. Leaders should have a vision, accompanied by a strategic plan, advice on resources and good communication with professionals working at a local level. This requires both championship over a number of years within the relevant professional organisations, with local leaders advocating for the service with commissioners and other local providers. The vision must be based on the best possible evidence and have the support of a wide range of stakeholders, along with clear benefits for patients, if it is to be successful.

Workforce planning is crucial for ongoing service delivery. The first consultant community paediatricians were generally appointed to replace retiring Senior Clinical Medical Officers who had transferred from the local authority to the health service. While this is an exceptional evolutionary course within the NHS establishing the role of a new consultant, complete with model job descriptions was an essential part of national workforce planning with the Joint Committee for Higher Professional Training. The development of special interest groups or specialty groups within Royal Colleges can play an important role in professional cohesion and direction. The British Association for Community Child Health (BACCH) evolved from the Community Paediatric Group in the same way that the Community Geriatrics Special Interest Group evolved from the Primary and Continuing Care Special-Interest Group. Such groups can provide a forum for the discussion of difficult issues, provide advice on workforce and training, organize academic meetings and advocate for the development of their specialty.

Once a new role is agreed, estimates of the numbers of trainees and a training programme can be developed by the professional college to ensure new consultants have the appropriate competences. Numbers are likely to evolve over time as teams mature and potential subspecialties arise. This process of workforce planning and training must also keep an eye on the future to determine how new interventions are emerging as effective clinical practice, along with policy directions of the health service and the demographics and epidemiology of the population. The national development of children's palliative care teams enabled nursing staff to join health visitors and school nurses working in the community, broadening the range of nursing competences in the community child health team. The learning from community child health is that it is often difficult to introduce new models of service delivery without appropriate evidence of effectiveness and value, based on research. Operational research about new models of care, is both difficult to fund, design and evaluate and is further compounded by lack of academic research centers. Investment in health services research is essential for the long-term viability of any service. The publication of the results of large-scale quality improvement projects goes some way towards broadening the evidence base.

\section{Conclusion}

This personal review of the development of community child health services over last 30 years has highlighted a number of issues regarding clinical team development in community settings. Community geriatric training has been established more recently and consultant community geriatricians will be facing the practical issues of developing and embedding a new service. We have highlighted the importance of national leadership and policy to support the development of community-based geriatric services, especially for workforce estimates and developing training capacity. This has been complemented by newly appointed geriatricians acting as local champions to successfully negotiate the implementation, development and improvement of local teams. One of the challenges is enable traditional practice to evolve and additional change management training may be required to overcome some of the barriers which currently exist. Our experience of the development of community child health has been positive for children and families and we hope that some of our reflections may be helpful in the process of developing better services and outcomes for elderly people.

\section{References}

1. Barton A, Mulley G (2003) History of the development of geriatric medicine in the UK. Postgraduate Medical Journal. Postgrad Med J 79(930): 229-234.

2. Craft A, Dodd K. From an Association to a Royal College: The History of the British Paediatric Association and Royal College of Paediatrics and Child Health 1988-2016 Springer International Publishing AG.

3. Council of Europe (2011) Council of Europe guidelines on child friendly health care.

4. Regional Geriatric Program (RGP) of Toronto (2017) The senior friendly care framework

5. Department of Health (2001) National service framework for the older person. DH, London, UK.

6. Department of Health. National service framework for children and young people.

7. Oliver D, Foot C, Humphries R (2014) Making our health and care systems fit for an ageing population. The Kings Fund, UK.

8. Kossarova L (2015) 'Children's health: Seen but not heard?' Nuffield trust comment.

9. Viner R, Ward J, Cheung R, Wolfe I Hargreaves I (2018) Child health in 2030 in England: Comparisons with other wealthy countries, RCPCH.

10. Fabbri E, Zoli M, Gonzalez Freire M, Salive ME, Studenski SA, et al. (2015) Aging and multimorbidity: New tasks, priorities, and frontiers for integrated gerontological and clinical research. J Am Med Dir Assoc 16(8): 640-647.

11. NICE (2016) Multimorbidity: Clinical assessment and management. NICE guideline [NG56]. 
12. Kingston A, Robinson L, Booth H, Knapp M, Jagger C (2018) Projections of multi-morbidity in the older population in England to 2035: Estimates from the Population Ageing and Care Simulation (PACSim) model. Age and Ageing 47(3): 374-380.

13. Mc Guinness F (2018) Poverty in the UK: Statistics. House of commons briefing paper. Number 7096.

14. Age UK (2017) Factsheet 78. Safeguarding older people from abuse and neglect.

15. NHS England North (2014) Safeguarding adults.

16. HM Government (2018) Working together to safeguard children. A guide to inter-agency working to safeguard and promote the welfare of children.

17. (2016) Public Health England The NHS Atlas of variation in healthcare reducing unwarranted variation to increase value and improve quality.
18. Metze RN, Abma TA, Kwekkeboom MH (2018) Family group conferencing for older adults: Social workers' views. J Social Work.

19. NHS England (2016) National quality board. How to ensure the right people, with the right skills, are in the right place at the right time. A guide to nursing, midwifery and care staffing capacity and capability

20. Rosenwax L, Spilsbury K, Arendts G, McNamara B, Semmens J (2015) Community-based palliative care is associated with reduced emergency department use by people with dementia in their last year of life: A retrospective cohort study. Palliat Med 29(8): 727-736.

21. The Kings Fund (2014) Imperial child health general practice hubs.

22. BACCH (2014) The Family Friendly Framework.

23. (2014) NHS England Practical guidance for commissioners, providers and nursing, medical and allied health professional leaders.

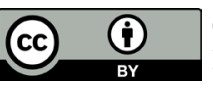

Creative Commons Attribution 4.0

International License

For possible submissions Click Here
Submit Article

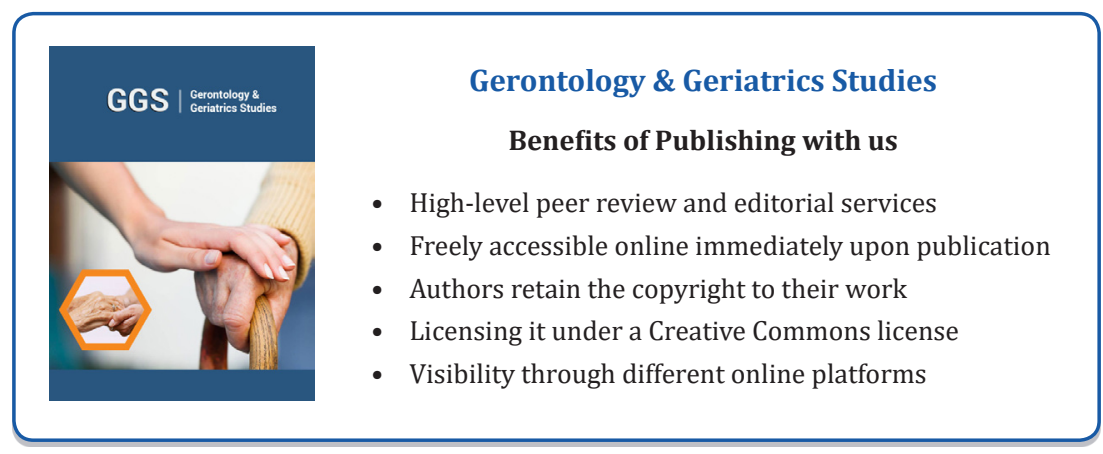

